\title{
Sexual quality of life assessment in young women with breast cancer during adjuvant endocrine therapy and patient-reported supportive measures
}

\author{
Angelique Bobrie ${ }^{1,2} \cdot$ Marta Jarlier $^{3} \cdot$ Aurore Moussion $^{4} \cdot$ William Jacot $^{1,2} \cdot$ Veronique D'Hondt $^{1,2}(0$
}

Received: 26 August 2021 / Accepted: 3 January 2022 / Published online: 14 January 2022

(c) The Author(s) 2022

\begin{abstract}
Purpose Sexual quality of life (QoL) is affected during and after breast cancer (BC) treatment. The purpose was to investigate sexual and global QoL and patient-reported measures to address this issue in young women ( $<51$ years) with BC after the acute treatment phase, during adjuvant endocrine therapy.

Methods Three EORTC questionnaires and an additional specific questionnaire, developed for the study, were used to assess sexual and global QoL and patient-reported supportive measures in BC patients who had received their endocrine therapy for at least 24 months. Among the 54 eligible patients, 45 (83\%) agreed to participate in the study.

Results We showed a deterioration in sexual QoL and poor communication with healthcare professionals. Most patients (88.9\%) declared that it was important that sexuality should be discussed with caregivers and that the partner should also be involved. Most patients (60\%) had taken at least one action to overcome their sexual problems. Most of these interventions (63\%) originated from the patient herself.

Conclusions Sexual QoL is a major issue in young BC patients and is poorly addressed by healthcare professionals. Most of the supportive methods used by the patients to overcome these side effects were on their own initiative. Communication and counseling on sexuality by healthcare professionals need to be improved during BC treatment. Patients suggested supportive measures they would find useful and appropriate to develop in the clinic. The final goal is to improve the sexual QoL of $\mathrm{BC}$ patients with the appropriate intervention and support.
\end{abstract}

Keywords Sexual quality of life $\cdot$ Patient-reported measures $\cdot$ Breast cancer $\cdot$ Supportive care $\cdot$ Survivorship

\section{Introduction}

Advances in $\mathrm{BC}$ diagnosis and treatment have led to the improvement of the patients' prognosis, with a 5-year survival in wealthier countries now reaching $90 \%$ [1]. The

Veronique D'Hondt

veronique.dhondt@icm.unicancer.fr

1 Medical Oncology Department, Institut du Cancer de Montpellier (ICM), 208 Avenue des Apothicaires, 34298 Montpellier Cedex5, France

2 Institut de Recherche en Cancerologie de Montpellier (IRCM), INSERM, University of Montpellier, Montpellier, France

3 Biometrics Unit, Institut du Cancer de Montpellier, Montpellier, France

4 Medical Research Unit, Institut du Cancer de Montpellier, Montpellier, France disease and its treatment can affect the day-to-day lives of $\mathrm{BC}$ survivors, and these physical, psychological, and social concerns become important issues to be addressed in clinic [2]. Among these QoL concerns, sexual difficulties have been more recently studied [3-7]. In this regard, a specific health-related quality of life questionnaire (HRQLQ) dedicated to evaluating sexual dysfunction related to the disease and its treatment, in cancer patients, the EORTC SHQ-22, has recently been developed [8].

We previously published the results of a longitudinal assessment analyzing the sexual and global QoL of 106 women with hormone receptor-positive $(\mathrm{HR}+) \mathrm{BC}$ during the first year of endocrine therapy, using the EORTC SHQ22 and global HRQLQ [9]. This study showed a deterioration of patients' sexual QoL as compared to women in the general population and a very low communication level about sexual themes between patients and healthcare givers. 
In this new study, we focused on young patients less than 51 years old, a population whose sexual $\mathrm{QoL}$ is more frequently and severely affected following BC care [9-13]. We analyzed the sexual and global QoL in women with $\mathrm{HR}+\mathrm{BC}$ who had received their endocrine therapy for at least 24 months, to analyze sexual dysfunction long after the acute surgical, chemotherapeutic, and radiotherapeutic treatment phases. We also used an additional specific questionnaire, proposed in the context of our study, to question women on the actions taken individually to address sexual issues and which supportive measures they would find useful and appropriate to develop in the clinic.

\section{Materials and methods}

\section{Study design and subject recruitment}

This unicentric prospective study (CUPIDON 2) was conducted at the Institut du Cancer de Montpellier (ICM). The protocol was approved by the French Ethics Committee and the internal review board of the institution. Patients received an information letter and all provided written consent before enrolment. The primary objective was to evaluate the sexual QoL of women younger than 51 years old with $\mathrm{HR}+\mathrm{BC}$ who had received their endocrine therapy for at least 24 months using the EORTC SHQ-22 questionnaire. The secondary objectives were: (1) to evaluate the global QoL at the same time-point using the EORTC QLQ-C30 [14] and QLQ-BR23 [15] questionnaires; (2) to evaluate the information received by patients about sexual issues; (3) to evaluate the need for specific management of sexual troubles; (4) to describe supportive measures or therapeutic interventions used by women on their own initiative using the specific Cupidon questionnaire, proposed for the study. Eligible women were between 18 and 51 years old, selfdeclared sexually active, displaying HR + early BC, and having completed surgery as well as chemotherapy and/or radiotherapy when indicated. They all had received their endocrine therapy for at least 24 months.

\section{Data and measures}

General health and socio-demographic information, including employment status, physical activity, and partner status, were self-reported by participants. Age, weight, size, menopausal status, pathology report, type of treatment (type of surgery, radiotherapy, and chemotherapy) as well as comorbidities were collected from the medical records.

Patients were invited to complete the three EORTC HRQLQ only once.

The general EORTC QLQ-C30 questionnaire dedicated to all cancer patients includes 30 items assessing the global health status with 5 functional scores (physical, role, cognitive, social, and emotional) and 9 symptom scores (nausea and vomiting, pain, fatigue, dyspnea, sleep disturbances, appetite loss, constipation, diarrhea, and financial difficulties).

The specific EORTC QLQ-BR23 module dedicated to breast cancer patients includes 23 items allowing to assess 4 functional scores (body image, sexual functioning, sexual enjoyment, and future perspective) and 4 symptom scores (systemic side effects, arm symptoms, breast symptoms, and being upset by hair loss).

The EORTC SHQ-22 is a multi-dimensional QoL instrument used to measure sexual health in patients with cancer (men or women). This new tool covers both sexual functioning and psychosexual components. It includes 8 items on sexual satisfaction, 3 items on sexual pain, and 11 single items in an integrative approach, leading to 7 functional scales and 4 symptom scales.

In these questionnaires, higher scores in the functioning scales indicate a better functional level, whereas higher scores in the symptom scales indicate the severity of problems. A user agreement for the use of the EORTC SHQ-22 was signed.

In addition, a homemade questionnaire containing 11 questions was proposed to patients in order to explore (1) the information regarding sexuality received during treatment, (2) the supportive measures or therapeutic interventions used by patients to overcome sexual dysfunction, and (3) the need and acceptability for specific management of sexual dysfunction. This specific Cupidon questionnaire is reproduced in extenso in the supplemental data.

\section{Statistical considerations}

In this descriptive study, the sample size was based on the recruitment capacity of our institution, which represented a potential of around 50 patients meeting the inclusion criteria of the study for the planned duration. Considering an $80 \%$ acceptance rate (20\% refusal), a total of 40 patients were expected to participate. The inclusion of at least 40 patients permits to estimate (for descriptive purposes) the mean scores of the dimensions of the EORTC SHQ-22 (ranging from 0 to 100) and its $95 \%$ confidence intervals (95\% CI) with a width of 15 and hypothesizing a standard deviation of 24 [16].

Scorings of the three EORTC HRQLQ were calculated according to the EORTC Scoring Manuals, and results were presented as means and standard deviations (SD). Categorical variables were described using frequencies and percentages.

Statistical analysis was performed using the Stata v16 software (College Station, TX, 2019, USA). 


\section{Results}

\section{Patients' characteristics}

Among the 65 patients screened during consultations between February and April 2020, to participate in the study, 11 were not eligible ( 8 had no sexual activity, 2 had relapsed, 1 had discontinued her endocrine therapy), and 9 refused to participate. A total of 45 patients have been included and completed the four questionnaires. Clinical and socio-demographic characteristics are summarized in Table 1 . The median age was 45 years. A majority of women had been treated by breast conserving surgery (62\%), and had received chemotherapy (87\%). Most women $(87 \%)$ had received endocrine treatment for 2 to 5 years. Sixty percent of women were premenopausal at cancer diagnosis. Endocrine therapy consisted mostly of tamoxifen (73\%), and included a chemical castration in $18 \%$ of cases. According to the body mass index (BMI), 33 and $11 \%$ of patients were overweight or obese, respectively. Most patients (58\%) practiced regular physical activity, i.e., minimum of $30 \mathrm{~min}, 3$ times a week, and $76 \%$ were professionally active. All women were sexually active at baseline (inclusion criteria) and $17 \%$ declared a previous history of sexual problems either in the couple $(5 \%)$ or of their partner $(12 \%)$.

\section{Sexual health questionnaire}

Mean scores for the EORTC SHQ-22 questionnaire are shown in Table 2. The importance attributed to sexual activity appeared relatively preserved (mean score of 60.00), but sexual satisfaction was lower (mean score of 45.80). The libido and the impact of treatment on sexual life scores were low (mean scores of 29.63 and 37.78) and the communication about sexuality with professionals score was extremely low (mean score of 11.11). The feeling of security with the partner and the femininity were less altered (mean scores of 52.71 and 59.69 respectively).

Vaginal dryness was the most important symptom (a mean score of 57.66). Fatigue appeared important (mean score of 49.24) as well as pain related to intercourses (a mean score of 31.98). Worrying about incontinence was rare (mean score 9.09).

\section{Quality of life questionnaires}

Mean scores for the EORTC QLQ-C30 and the EORTC QLQ-BR23 are shown in Table 3.

According to the EORTC QLQ-C30, the global health status score was 69.63 . The mean scores of
Table 1 Clinical and socio-demographic characteristics of the women

\begin{tabular}{|c|c|}
\hline & Total $(n=45)$ \\
\hline Median age-years (range) & $45(33-50)$ \\
\hline \multicolumn{2}{|l|}{ Surgery } \\
\hline Breast conserving & $28(62 \%)$ \\
\hline Mastectomy & $17(38 \%)$ \\
\hline \multicolumn{2}{|l|}{ Adjuvant chemotherapy } \\
\hline Yes & $39(87 \%)$ \\
\hline No & $6(13 \%)$ \\
\hline \multicolumn{2}{|l|}{ Adjuvant radiotherapy } \\
\hline Yes & $41(91 \%)$ \\
\hline No & $4(9 \%)$ \\
\hline \multicolumn{2}{|l|}{ Adjuvant endocrine therapy } \\
\hline Tamoxifen & $33(73 \%)$ \\
\hline Tamoxifen followed by LH-RH agonist + AI & $5(11 \%)$ \\
\hline Tamoxifen followed by AI & $4(9 \%)$ \\
\hline LH-RH agonist + AI & $3(7 \%)$ \\
\hline \multicolumn{2}{|l|}{ Time since the beginning of the endocrine therapy } \\
\hline $2-5$ years & $39(87 \%)$ \\
\hline$>5$ years & $6(13 \%)$ \\
\hline \multicolumn{2}{|l|}{ Menopausal status at cancer diagnosis } \\
\hline Premenopausal & $27(60 \%)$ \\
\hline Postmenopausal & $18(40 \%)$ \\
\hline \multicolumn{2}{|l|}{ Body mass index } \\
\hline Median & 23.6 \\
\hline$<18$ & $3(7 \%)$ \\
\hline $18-24$ & $22(49 \%)$ \\
\hline $25-29$ & $15(33 \%)$ \\
\hline$\geq 30$ & $5(11 \%)$ \\
\hline \multicolumn{2}{|l|}{ Children } \\
\hline Yes & $38(84 \%)$ \\
\hline No & $7(16 \%)$ \\
\hline \multicolumn{2}{|l|}{ Physical activity } \\
\hline Yes & $26(58 \%)$ \\
\hline No & $19(42 \%)$ \\
\hline \multicolumn{2}{|l|}{ Smoker } \\
\hline Yes & $14(31 \%)$ \\
\hline No & $16(36 \%)$ \\
\hline Stopped & $15(33 \%)$ \\
\hline \multicolumn{2}{|l|}{ Professional activity } \\
\hline Worker & $34(76 \%)$ \\
\hline Unemployed & $10(22 \%)$ \\
\hline Retired & $1(2 \%)$ \\
\hline \multicolumn{2}{|l|}{ History of sexual problem } \\
\hline Within the current couple & $2(5 \%)$ \\
\hline Of the partner & $5(12 \%)$ \\
\hline
\end{tabular}

Abbreviations: AI, aromatase inhibitor; $L H-R H$, luteinizing hormonereleasing hormone 
Table 2 Sexual quality of life assessed by the EORTC SHQ-22

\begin{tabular}{lrrr}
\hline EORTC SHQ-C22 & \multicolumn{3}{l}{$N=45$} \\
\cline { 2 - 4 } & $N$ & Mean & SD \\
\hline Functional scales & 45 & 45.80 & 22.89 \\
$\quad$ Sexual satisfaction & 45 & 60.00 & 34.52 \\
$\quad$ Importance of sexual activity & 45 & 29.63 & 33.50 \\
Libido & 45 & 37.78 & 39.31 \\
Impact of treatment on sexual life & 45 & 11.11 & 23.57 \\
Communication with professionals & 43 & 52.71 & 40.00 \\
$\quad$ Security with partner & 43 & 59.69 & 42.14 \\
$\quad$ Femininity & & & \\
Symptom scales & 41 & 31.98 & 32.03 \\
$\quad$ Sexual pain & 44 & 9.09 & 24.23 \\
$\quad$ Worrying about incontinence & 44 & 49.24 & 39.69 \\
$\quad$ Fatigue & 37 & 57.66 & 42.05 \\
$\quad$ Vaginal dryness & &
\end{tabular}

$N$, number of women who answered the question. Higher scores in the functioning scales indicate better functional level whereas, higher scores in the symptom scales indicate the severity of the symptoms

physical functioning, role functioning, and social functioning appeared better than those of emotional and cognitive functioning.

Regarding the symptom scales, the highest scores were fatigue and insomnia (mean scores of 41.48 and 46.67, respectively).

According to the EORTC QLQ-BR23, the score for the body image was 66.30 , while the score for the sexual functioning scale appeared low (a mean score of 35.19). Sexual enjoyment appeared better (a mean score of 62.75), but only $75 \%$ of patients answered this item. The side effects of systemic therapy and local breast and arm symptoms had similar scores. With a minimum of 2 years since the end of the chemotherapy, women were no more concerned by hair loss.

\section{Specific Cupidon questionnaire}

The answers to the specific Cupidon questionnaire are shown in Table 4.

Most patients (73.3\%) had not received or did not remember receiving any information about sexuality from their oncologist or any other caregiver. Among the 12 patients who had received information, $10(83.3 \%)$ were satisfied with the received information. Most patients $(86.7 \%)$ had never discussed potential sexual problems with their oncologist or caregiver.

When asked why they did not discuss about sex with them, most patients declared having no question about sexuality (69.2\%, agree or fully agree) and feeling it was a minor issue as compared to cancer and the cancer treatment $(66.7 \%)$. Only a minority of patients felt that the caregivers
Table 3 Global quality of life assessed by the EORTC QLQ-C30 and the EORTC QLQ-BR23

\begin{tabular}{|c|c|c|c|}
\hline & \multicolumn{3}{|c|}{$N=45$} \\
\hline & $\mathrm{N}$ & Mean & SD \\
\hline \multicolumn{4}{|l|}{ EORTC QLQ-C30 } \\
\hline \multicolumn{4}{|l|}{ Functional scales } \\
\hline Global health status & 45 & 69.63 & 17.60 \\
\hline Physical functioning & 45 & 87.85 & 12.89 \\
\hline Role functioning & 45 & 82.22 & 24.46 \\
\hline Emotional functioning & 45 & 55.37 & 32.18 \\
\hline Cognitive functioning & 45 & 59.26 & 31.89 \\
\hline Social functioning & 45 & 80.37 & 23.65 \\
\hline \multicolumn{4}{|l|}{ Symptom scales } \\
\hline Fatigue & 45 & 41.48 & 27.67 \\
\hline Nausea and vomiting & 45 & 4.07 & 9.51 \\
\hline Pain & 45 & 27.04 & 29.15 \\
\hline Dyspnea & 45 & 22.96 & 24.44 \\
\hline Insomnia & 45 & 46.67 & 43.46 \\
\hline Appetite loss & 45 & 7.41 & 21.19 \\
\hline Constipation & 45 & 11.85 & 22.65 \\
\hline Diarrhea & 45 & 8.89 & 16.51 \\
\hline Financial difficulties & 45 & 13.33 & 31.30 \\
\hline \multicolumn{4}{|l|}{ EORTC QLQ-BR23 } \\
\hline \multicolumn{4}{|l|}{ Functional scales } \\
\hline Body image & 45 & 66.30 & 30.25 \\
\hline Sexual functioning & 45 & 35.19 & 24.68 \\
\hline Sexual enjoyment & 34 & 62.75 & 28.15 \\
\hline Future Perspective & 45 & 40.74 & 31.69 \\
\hline \multicolumn{4}{|l|}{ Symptom scales } \\
\hline Systemic therapy side effects & 45 & 26.56 & 17.69 \\
\hline Breast symptoms & 44 & 22.73 & 18.97 \\
\hline Arm symptoms & 44 & 22.73 & 23.96 \\
\hline Upset by hair loss & 0 & & \\
\hline
\end{tabular}

$N$, number of women who answered the question

were too busy to discuss (25.7\%) or felt discomfort (30.8\%) to talk about the subject.

At the same time, most patients $(88.9 \%)$ declared it important that sexuality and its potential problems should be discussed with caregivers and that the partner should be involved in the discussion $(73.4 \%)$. Half of the patients (53.3\%) wished for a systematic initial consultation with a sexologist, and $75.6 \%$ wished that such a consultation would be available when needed.

The majority of patients $(60 \%)$ had tried at least one method to overcome their sexual problems, in order of frequency: local treatment like vaginal moisturizer (48.9\%), consultation with a psychologist (17.8\%), while a consultation with a sexologist remained rare (2.2\%). Most of these interventions (63\%) originated from the patient herself. Eight patients declared having used other help: consultation 
Table 4 Patient-reported communication with health professionals and supportive measures and patients' suggestions for sexual care, assessed by the CUPIDON questionnaire

Evaluation of the received information and satisfaction $\quad N=45 \%$

1. Did you receive any information from the medical oncologist or another caregiver on the possible impacts of cancer and of your treatment on sexuality?

\begin{tabular}{|c|c|}
\hline No & 24 \\
\hline Yes $^{1}$ & 12 \\
\hline I don't remember & 9 \\
\hline mong the 12 patients: 10 we & \\
\hline 'm satisfied with the inform & \\
\hline Strongly disagree/disagree & 14 \\
\hline No opinion & 19 \\
\hline Agree/fully agree & 12 \\
\hline
\end{tabular}

3. Have you had any discussion with the oncologist or another caregiver from the Cancer Center about potential sexual problems?

No

Yes

Talking about sexual health and barriers

4. I did not discuss about sexuality because:

I had no question or I didn't feel the need to discuss about it

Strongly disagree/disagree

No opinion

Agree/fully agree

I considered sexuality as a minor issue compared to cancer treatment

Strongly disagree/disagree

No opinion

Agree/fully agree

Physicians and/or nurses looked in a hurry or too busy

Strongly disagree/disagree

No opinion

Agree/fully agree

I felt too shy and/or discomfort to discuss this topic

Strongly disagree/disagree

No opinion

Agree/fully agree

I felt discomfort since my physician was a man and/or since I had no positive contact with him/her

Strongly disagree/disagree

No opinion

Agree/fully agree

It is in contradiction with my education, beliefs, culture

Strongly disagree/disagree

No opinion

Agree/fully agree

Management of sexual issues: evaluation of the needs in information and therapeutic proposals

5. In my opinion, it is important that a physician and/or a caregiver from the Cancer Center addresses the issue of sexuality and its potential dysfunction due to the cancer or to cancer treatments

$\begin{array}{ll}\text { Strongly disagree/disagree } & 1 \\ \text { No opinion } & 2.2 \\ \text { Agree/fully agree } & 8.9 \\ \text { P } & 88.9\end{array}$

6. In my opinion, it is important that a physician and/or a caregiver from the Cancer Center addresses the issue of sexuality and its potential dysfunction due to the cancer or to cancer treatments with my partner 
Table 4 (continued)

Evaluation of the received information and satisfaction

$N=45 \%$

Agree/fully agree

No partner

7. I would have wished that a consultation about sexuality was systematically proposed at the beginning of cancer treatment

Strongly disagree/disagree

No opinion

Agree/fully agree

8. I would have wished that a consultation about sexuality was available in the Cancer Center when needed during my treatment Strongly disagree/disagree

No opinion

$12 \quad 26.7$

$9 \quad 20.0$

$24 \quad 53.3$

Agree/fully agree

24.4

$9 \quad 20.0$

$34 \quad 75.6$

9. Have you been using any of these methods for sexual purpose since the beginning of your disease? (each sub-item was binary, only category ' yes' is shown)

Consultation with a psychologist

Consultation with a psychiatrist

Consultation with a sexologist

Pelvic floor physical therapy

Use of vaginal moisturizer

Use of vaginal laser therapy

Use of at least one the methods mentioned above

10. If you have been using any method from the previous question, have you decided it on your own?

Yes (own initiative)

Yes (own initiative) and advice (from my: oncologist $(n=1)$, my gynecologist $(\mathrm{n}=1))$

No: advice ${ }^{2}$

${ }^{2}$ from: oncologist $(n=1)$, general practitioner $(n=2)$, gynecologist $(n=5)$

\section{Patients' suggestions for sexual care}

In your opinion, which method would be useful and/or appropriate to address the sexual problems (several answers possible)?

Consultation with a caregiver trained in sexology

Strongly disagree/disagree

No opinion

Agree/fully agree

Consultation with a psychiatrist

Strongly disagree/disagree

No opinion

Agree/fully agree

Consultation with a sexologist

Strongly disagree/disagree

No opinion

Agree/fully agree

Couple consultation

Strongly disagree/disagree

13.3

920.0

$30 \quad 66.7$

No opinion

6

13.3

Agree/fully agree

Group consultation

Strongly disagree/disagree

No opinion

Agree/fully agree

On-line therapy using on-line questionnaires and consultations

Strongly disagree/disagree 
Table 4 (continued)

\begin{tabular}{lc}
\hline Evaluation of the received information and satisfaction & $N=45$ \\
\hline Agree/fully agree & $\%$ \\
Pelvic floor physical therapy & 46.7 \\
Strongly disagree/disagree & 21 \\
No opinion & 4.4 \\
Agree/fully agree & 22.2 \\
Vaginal moisturizer & 73.4 \\
Strongly disagree/disagree & 10 \\
No opinion & 33 \\
Agree/fully agree & 2.2 \\
\hline
\end{tabular}

$N$, number of women who answered the question

with a gynecologist $(n=5)$, with a general practitioner $(n=2)$, and with a midwife $(n=1)$.

Patients suggested as desirable the following interventions in order of frequency: consultation with a caregiver trained in sexology (91.1\%), vaginal moisturizer $(77.8 \%)$, pelvic floor physical therapy $(73.4 \%)$, combined consultation with her partner and a caregiver (71.1\%), consultation with a sexologist (66.7\%), online psychotherapy (46.7\%), psychiatric consultation (40\%), and group consultation (24.4\%).

\section{Discussion}

This study described the sexual and global QoL of 45 young $\mathrm{BC}$ women during adjuvant endocrine treatment for at least 24 months, as well as the actions taken and suggested by these women to overcome the encountered problems.

First of all, women displayed a high level of interest in the study, as demonstrated by the $83 \%$ participation rate and a high rate of response to the items of the questionnaire.

The scores observed for the sexual QoL evaluation with the EORTC SHQ-22 were similar to those previously observed using the same questionnaire in a population without age restriction [9] during endocrine therapy (sexual satisfaction scores were 43.43 and 45.80 in the previous and current studies, respectively), with, though, a higher importance for sexual activity in this younger population (scores of 42.95 and 60.00 , respectively). The observed score deteriorations were similar in both studies, with an extremely low score for communication with professionals and a high level of alteration of the sexual satisfaction and libido scores. This deleterious effect of $\mathrm{BC}$ and its treatment on sexual QoL is consistent with what has been shown in the literature with other questionnaires [17-21] and is significant when compared to the French general population, in which the rate of sexual satisfaction of women was $88 \%$ in a 2006 survey including 6824 women [22]. It should be noted that the professional activity, physical activity, and body mass index rates of our study population were similar to those of the same-aged general population [23, 24].

The global QoL deterioration analyzed with the QLQC30 and BR23 was also similar to what we previously reported [9], with the most frequent symptoms being fatigue and insomnia.

We explored the actions taken and proposed by patients to overcome these sexual dysfunctions using a homemade questionnaire specific to our study. Firstly, most patients had not received any information; either a fortiori help from their oncologist nor any caregiver. A vast majority of them declared that is was important that sexuality should be discussed and that their partner should be involved. However, among the women who had received information from their oncologist or any caregiver, the vast majority were satisfied with the received information, which is altogether encouraging, since these caregivers were not trained before the study.

The majority of patients used interventions to overcome sexual problems on their own initiative, underlying the need for specific management of this issue. They stated, mostly not based on their personal experience, that it would be desirable to develop, by order of frequency: consultation with a caregiver trained in sexology, vaginal moisturizer, pelvic floor physical therapy, consultation with their partner, consultation with a sexologist, and less frequently, any kind of psychotherapy.

Most patients declared that sexuality remains a minor issue compared to cancer treatment as the main reason to explain why they did not discuss it with their oncologist or caregiver. This seemingly contradictory response might reflect the patients' ambivalence about the theme and/or the fact that they want to prioritize the time with their oncologist to focus on the medical information regarding the cancer and its treatment. The role of the oncologist is probably mostly to raise the subject with the patient and question the need for such a dedicated consultation.

The main limitations of our study are the low number of patients included and the absence of longitudinal evaluation. 
Moreover, a memory bias might have altered the content of the reported events when patients were asked about their history of sexual problems. The strengths are the homogeneity of this young women's population as well as the use of standardized EORTC questionnaires integrating physical, psychological, and social aspects to evaluate sexual QoL. Moreover, despite the fact that the specific Cupidon questionnaire is not a validated research instrument, asking women their opinion on the actions that could be useful and appropriate to develop in clinic is crucial. This latter information from the involved women going through this issue is precious and should be used to identify the actions to be taken to support these women. The key proposals from the affected patients should be considered to tailor the patient's management, with the final goal being to improve the QoL of our patients with adequate intervention and support.

Supplementary Information The online version contains supplementary material available at https://doi.org/10.1007/s00520-022-06810-3.

Acknowledgements The authors would like to thank all the women who participated in the study.

\begin{abstract}
Author contribution Angelique Bobrie participated in conducting the study, including the patients, collecting the data, and writing the manuscript. Marta Jarlier participated in the statistical analysis and in writing the manuscript. Aurore Moussion participated in the construction of the study and in writing the manuscript. William Jacot participated in the construction of the study and in writing the manuscript. Véronique D'Hondt participated in the construction of the study, conducting the study, and writing the manuscript.
\end{abstract}

Data availability The data that support the findings of this study are available from the authors upon reasonable request.

Code availability Statistical code can be made available on written request to the corresponding author.

\section{Declarations}

Ethics approval The protocol was approved by the French Ethics Committee and the internal review board of the institution. CPP $\mathrm{N}^{\circ}$ 2019/100 - promoter ref 19.12.09.63035.

Consent to participate All patients received an information letter and all provided written consent before the enrolment.

Consent for publication Not applicable.

Conflict of interest The authors declare no competing interests.

Open Access This article is licensed under a Creative Commons Attribution 4.0 International License, which permits use, sharing, adaptation, distribution and reproduction in any medium or format, as long as you give appropriate credit to the original author(s) and the source, provide a link to the Creative Commons licence, and indicate if changes were made. The images or other third party material in this article are included in the article's Creative Commons licence, unless indicated otherwise in a credit line to the material. If material is not included in the article's Creative Commons licence and your intended use is not permitted by statutory regulation or exceeds the permitted use, you will need to obtain permission directly from the copyright holder. To view a copy of this licence, visit http://creativecommons.org/licenses/by/4.0/.

\section{References}

1. Allemani C, Matsuda T, Di Carlo V et al (2018) Global surveillance of trends in cancer survival 2000-14 (CONCORD-3): analysis of individual records for 37513025 patients diagnosed with one of 18 cancers from 322 population-based registries in 71 countries. Lancet 391:1023-1075. https://doi.org/10.1016/S01406736(17)33326-3

2. Runowicz CD, Leach CR, Henry NL et al (2016) American Cancer Society/American Society of Clinical Oncology Breast Cancer Survivorship Care Guideline. J Clin Oncol 34:611-635. https:// doi.org/10.1200/JCO.2015.64.3809

3. Ananth H, Jones L, King M, Tookman A (2003) The impact of cancer on sexual function: a controlled study. Palliat Med 17:202205. https://doi.org/10.1191/0269216303pm759oa

4. Krychman ML, Katz A (2012) Breast cancer and sexuality: multimodal treatment options (CME). J Sex Med 9:5-13. https://doi. org/10.1111/j.1743-6109.2011.02566.x

5. Frechette D, Paquet L, Verma S et al (2013) The impact of endocrine therapy on sexual dysfunction in postmenopausal women with early stage breast cancer: encouraging results from a prospective study. Breast Cancer Res Treat 141:111-117. https://doi.org/ 10.1007/s10549-013-2659-y

6. Aerts L, Christiaens MR, Enzlin P et al (2014) Sexual functioning in women after mastectomy versus breast conserving therapy for early-stage breast cancer: a prospective controlled study. Breast 23:629-636. https://doi.org/10.1016/j.breast.2014.06.012

7. Pup LD, Villa P, Amar ID et al (2019) Approach to sexual dysfunction in women with cancer. Int J Gynecol Cancer 29. https:// doi.org/10.1136/ijgc-2018-000096

8. Oberguggenberger AS, Nagele E, Inwald EC et al (2018) Phase 1-3 of the cross-cultural development of an EORTC questionnaire for the assessment of sexual health in cancer patients: the EORTC SHQ-22. Cancer Med 7:635-645. https://doi.org/10.1002/cam4. 1338

9. Aptecar L, Fiteni F, Jarlier M et al (2021) Prospective evaluation of sexual health in breast cancer women during the first year of adjuvant hormonal treatment using a cancer patient's dedicated questionnaire: a glaring gap of communication between health professionals and patients. Breast Cancer Res Treat. https://doi. org/10.1007/s10549-020-06062-x

10. Fobair P, Stewart SL, Chang S et al (2006) Body image and sexual problems in young women with breast cancer. Psychooncology 15:579-594. https://doi.org/10.1002/pon.991

11. Muñoz M (2010) Quality of life during treatment in young women with breast cancer. Breast Cancer Res Treat 123:75-77. https:// doi.org/10.1007/s10549-010-1061-2

12. Howard-Anderson J, Ganz PA, Bower JE, Stanton AL (2012) Quality of life, fertility concerns, and behavioral health outcomes in younger breast cancer survivors: a systematic review. J Natl Cancer Inst 104:386-405. https://doi.org/10.1093/jnci/djr541

13. Hsu T, Ennis M, Hood N et al (2013) Quality of life in long-term breast cancer survivors. J Clin Oncol Off J Am Soc Clin Oncol 31:3540-3548. https://doi.org/10.1200/JCO.2012.48.1903

14. Aaronson NK, Ahmedzai S, Bergman B et al (1993) The European Organization for Research and Treatment of Cancer QLQ-C30: a quality-of-life instrument for use in international clinical trials 
in oncology. JNCI J Natl Cancer Inst 85:365-376. https://doi.org/ 10.1093/jnci/85.5.365

15. Sprangers MA, Groenvold M, Arraras JI et al (1996) The European Organization for Research and Treatment of Cancer breast cancer-specific quality-of-life questionnaire module: first results from a three-country field study. J Clin Oncol 14:2756-2768. https://doi.org/10.1200/JCO.1996.14.10.2756

16. 9781405146500: sample size tables for clinical studies - AbeBooks - Machin, David; Campbell, Michael J.; Tan, Say-Beng; Tan, Sze-Huey: 1405146508. https://www.abebooks.fr/97814 05146500/Sample-Size-Tables-Clinical-Studies-1405146508/ plp. Accessed 2 Nov 2020

17. Ribi K, Luo W, Walley BA et al (2020) Treatment-induced symptoms, depression and age as predictors of sexual problems in premenopausal women with early breast cancer receiving adjuvant endocrine therapy. Breast Cancer Res Treat 181:347-359. https:// doi.org/10.1007/s10549-020-05622-5

18. Den Oudsten BL, Van Heck GL, Van der Steeg AFW et al (2010) Clinical factors are not the best predictors of quality of sexual life and sexual functioning in women with early stage breast cancer. Psychooncology 19:646-656. https://doi.org/10.1002/pon.1610

19. Yang EJ, Kim S-W, Heo C-Y, Lim J-Y (2011) Longitudinal changes in sexual problems related to cancer treatment in Korean breast cancer survivors: a prospective cohort study. Support Care Cancer 19:909-918. https://doi.org/10.1007/s00520-010-0885-y
20. Rosenberg SM, Tamimi RM, Gelber S et al (2014) Treatmentrelated amenorrhea and sexual functioning in young breast cancer survivors: sexual Function and Breast Cancer. Cancer 120:22642271. https://doi.org/10.1002/cncr.28738

21. Kowalczyk R, Nowosielski K, Cedrych I et al (2019) Factors affecting sexual function and body image of early-stage breast cancer survivors in Poland: a short-term observation. Clin Breast Cancer 19:e30-e39. https://doi.org/10.1016/j.clbc.2018.09.006

22. Bajos N, Bozon M, Beltzer N, l'équipe CSF (2008) Sexualité, prévention et rapports sociaux de sexe au fil de la vie. Médecine/ sciences 24:24-32. https://doi.org/10.1051/medsci/2008242s24

23. Institut national de la statistique et des études économiques (France) (2020) Tableaux de l'économie française

24. DGOS (2021) Obésité : prévention et prise en charge. In: Ministère Solidar. Santé. https://solidarites-sante.gouv.fr/syste me-de-sante-et-medico-social/strategie-nationale-de-sante/prior ite-prevention-rester-en-bonne-sante-tout-au-long-de-sa-vie11031/priorite-prevention-les-mesures-phares-detaillees/article/ obesite-prevention-et-prise-en-charge. Accessed 27 Apr 2021

Publisher's note Springer Nature remains neutral with regard to jurisdictional claims in published maps and institutional affiliations. 